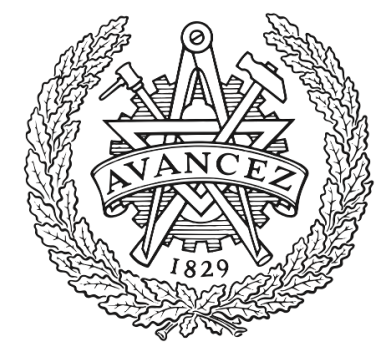

CHALMERS

UNIVERSITY OF TECHNOLOGY

\title{
Cosmic string and black hole limits of toroidal Vlasov bodies in general relativity
}

Downloaded from: https://research.chalmers.se, 2023-04-26 15:17 UTC

Citation for the original published paper (version of record):

Ames, E., Andreasson, H., Logg, A. (2019). Cosmic string and black hole limits of toroidal Vlasov bodies in general relativity. Physical Review D, 99(2).

http://dx.doi.org/10.1103/PhysRevD.99.024012

N.B. When citing this work, cite the original published paper. 


\title{
Cosmic string and black hole limits of toroidal Vlasov bodies in general relativity
}

\author{
Ellery Ames, ${ }^{1, *}$ Håkan Andréasson, ${ }^{2, \dagger}$ and Anders Logg $2, \$$ \\ ${ }^{1}$ Department of Mathematics, KTH, S-10044 Stockholm, Sweden \\ ${ }^{2}$ Department of Mathematical Sciences, Chalmers University of Technology and University of Gothenburg, \\ S-41296 Göteborg, Sweden
}

(Received 11 April 2018; published 8 January 2019)

\begin{abstract}
We numerically investigate limits of a two-parameter family of stationary solutions to the EinsteinVlasov system. The solutions are toroidal and have nonvanishing angular momentum. As the parameters are tuned to more relativistic solutions (measured e.g., by an increasing redshift) we provide evidence for a sequence of solutions which approaches the extreme Kerr black hole family. Solutions with angular momentum larger than the square of the mass are also investigated, and in the relativistic limit the near-field geometry of such solutions is observed to become locally rotationally symmetric about the matter density. The existence of a deficit angle in these regions is investigated.
\end{abstract}

DOI: $10.1103 /$ PhysRevD.99.024012

\section{INTRODUCTION}

In a previous work [1], three different types of stationary solutions to the axially symmetric Einstein-Vlasov system were constructed numerically. These were disclike solutions, spindlelike solutions, and toroidal solutions. The main aim of the study [1] was to go beyond the analytic solutions which were obtained in $[2,3]$ as perturbations of spherically symmetric Newtonian solutions. In particular, a question that was raised in [3] was if there exist regular stationary solutions which contain ergoregions. This question was answered affirmatively in [1] where it was found that the most relativistic members of the family of toroidal solutions do contain ergoregions.

The presence of ergoregions suggests that one may be approaching a family of rotating black hole solutions, and one aim of the present study is to investigate whether one has a sequence of stationary solutions which have black hole limiting members. Such a quasistationary transition to black hole solutions does not occur in the spherically symmetric setting due to a Buchdahl bound, which for a body of mass $\mathcal{M}$ and radius $\mathcal{R}$ reads $2 \mathcal{M} / \mathcal{R}<8 / 9$, and which applies to large classes of matter models; cf. [4]. In this case there is a gap such that $2 \mathcal{M} / \mathcal{R}$ cannot approach 1 . However, if one allows for charge, a similar bound relating the mass, radius, and total charge is known [5], and in this case there is no gap; that is, a quasistationary transition to a Reissner-Nordström black hole could be possible. Indeed, such a limit to the extremal Reissner-Nordström black hole has been shown by

\footnotetext{
*ellery@kth.se

†hand@chalmers.se

*logg@ chalmers.se
}

Meinel and Hütten [6]. In the case where one has angular momentum, the model black hole solutions are the Kerr family, which are parametrized by the mass $\mathcal{M}$ and angular momentum $\mathcal{J}$ with the restriction $|\mathcal{J}| \leq \mathcal{M}^{2}$, where equality is achieved for the extremal Kerr solution. The question of a quasistationary transition of fluid bodies to extremal black holes has been investigated analytically in the case of disc solutions for dust by Meinel [7,8], where he shows that the extremal Kerr solution is the only admissible limit. More general dust solutions have been investigated numerically by Ansorg et al. [9-11], and in particular, their study includes families of toroidal bodies. Below, we provide evidence of a quasistationary transition to an extreme Kerr black hole for a class of rotating toroidal solutions to the Einstein-Vlasov system.

To this end we study a two-parameter family of solutions parametrized by $E_{0}$ and $L_{0}$. The black hole limit is approached by decreasing $E_{0}$ for a certain critical value of $L_{0}$. This critical solution sequence has the feature that the extremal black hole solution is approached from the stable side of the binding energy curve and while $|\mathcal{J}| \geq \mathcal{M}^{2}$. We are, however, not able to go all the way to the extreme black hole limit. At some point near the black hole limit, the code halts the approach to the black hole and instead changes course towards a distinct limit. Solutions on such paths appear to approach Dirac-type matter distributions in a thin ring.

The solutions on the thin-ring path have angular momentum which is larger than $\mathcal{M}^{2}$, and thus, due to angular momentum conservation, the solutions are stable against collapse to a Kerr black hole. The geometry of these solutions is investigated and it is discovered that extreme members of such solution paths exhibit a localized region 
near the matter distribution that is rotationally symmetric about the matter. Such a region is suggestive of a conical geometry characterized by a nonvanishing deficit angle over an open set of coordinate values. The deficit angles that we compute for the extremely thin toroidal solutions appear to approach those consistent with a localized conical region and a geometry similar to cosmic string models, where the matter is represented by Dirac-type sources and where the spacetime geometry is computed for such a given singular matter distribution [12-14].

Before presenting the outline of the paper, let us briefly comment on some previous numerical studies of the static and stationary Einstein-Vlasov system. In spherical symmetry the static Einstein-Vlasov system has the advantage that it is sufficient to solve an initial value problem for an ordinary differential equation (ODE) to construct solutions. The structure and properties of these solutions, which include thin shells and mass-radius spirals, are quite interesting; cf. $[15,16]$. In addition, in the spherically symmetric case, there exist static massless solutions; cf. [17,18]. Earlier results in axisymmetry are due to Shapiro and Teukolsky (and collaborators) who investigated the Einstein-Vlasov system numerically in a long series of papers spanning from 1987 to 1994 . Their main aim was to study the evolution problem in the axially symmetric case, but in their general program they also constructed stationary toroidal solutions in $[19,20]$, which are similar to those at the early stages of our solution sequences.

The outline of the paper is as follows. In Sec. II we review the formulation of the Einstein-Vlasov system, referring to [1] for more details. The results of numerically solving a boundary value problem for this system are presented in Sec. III. In particular we discuss the overall behavior of the parameter space (Sec. III A), as well as the black hole (Sec. III B) and cosmic string (Sec. III C) limits. We end the paper with a brief discussion and conclusions in Sec. IV.

\section{THE AXISYMMETRIC EINSTEIN-VLASOV SYSTEM}

\section{A. Equations}

The formulation and numerical solution of the equations closely follows that in [1], to which we refer the reader for details. We parametrize the metric as in Bardeen [21],

$g=-e^{2 \nu} d t^{2}+e^{2 \mu} d \rho^{2}+e^{2 \mu} d z^{2}+\rho^{2} B^{2} e^{-2 \nu}(d \varphi-\omega d t)^{2}$,

where the coordinates $(t, \varphi)$ are associated to the time and angular commuting Killing fields, respectively, and the metric fields $\nu, \mu, \omega, B$ depend only on $\rho \in[0, \infty)$ and $z \in(-\infty, \infty)$. Vlasov matter is modeled by a distribution function $f$, depending on spacetime coordinates $x=$ $(t, \rho, z, \varphi)$ and four-momenta $p$. The momenta are taken to lie in the mass shell $\mathcal{P}$, defined at each spacetime point $x$ as the subset of forward oriented vectors satisfying $g_{x}(p, p)=-m_{p}^{2}$, where $m_{p}$ is the particle rest mass. The particle mass is assumed the same for all particles, and we make the choice $m_{p}=1$. The distribution function is transported along the geodesic flow of the spacetime by the Vlasov equation, and the coupled Einstein-Vlasov system is closed through an energy-momentum tensor, which takes the form

$$
T_{i j}(x):=\int_{\mathbb{R}^{3}} p_{i} p_{j} f(x, p) \sqrt{-\operatorname{det} g} \frac{\mathrm{d} p^{1} \mathrm{~d} p^{2} \mathrm{~d} p^{3}}{-p_{0}} .
$$

To solve the coupled Einstein-Vlasov system we make an Ansatz that the Vlasov distribution depends on the phasespace coordinates $(x, p)$ only through the particle angular momentum

$$
L=(\rho B)^{2} e^{-2 \nu}\left(p^{3}-\omega p^{0}\right)=\rho B e^{-\nu} v^{3},
$$

and particle energy

$$
\begin{aligned}
E & =e^{2 \nu} p^{0}+\omega(\rho B)^{2} e^{-2 \nu}\left(p^{3}-\omega p^{0}\right) \\
& =e^{\nu} \sqrt{m_{p}^{2}+\sum_{i=1}^{3}\left(v^{i}\right)^{2}+\omega L,}
\end{aligned}
$$

where it is convenient to work in the orthonormal frame $v^{0}=e^{\nu} p^{0}, v^{1}=e^{\mu} p^{1}, v^{2}=e^{\mu} p^{2}, v^{3}=\rho B e^{-\nu}\left(p^{3}-\omega p^{0}\right)$. In general this Ansatz takes the form $f(x, p)=F(E, L)$, for a suitable function $F$. Since $E$ and $L$ are conserved along the geodesics traveled by the particles, this choice of distribution function ensures that the Vlasov equation is satisfied. As a result, the full Einstein-Vlasov system is reduced to an elliptic integro-differential system of equations for the metric fields,

$$
\begin{aligned}
\Delta \nu= & 4 \pi\left(\Phi_{00}+\Phi_{11}+\left(1+(\rho B)^{2} e^{-4 \nu} \omega^{2}\right) \Phi_{33}+2 e^{-4 \nu} \omega \Phi_{03}\right) \\
& -\frac{1}{B} \nabla B \cdot \nabla \nu+\frac{1}{2} e^{-4 \nu}(\rho B)^{2} \nabla \omega \cdot \nabla \omega \\
\Delta B & =8 \pi B \Phi_{11}-\frac{1}{\rho} \nabla \rho \cdot \nabla B \\
\Delta \mu= & -4 \pi\left(\Phi_{00}+\Phi_{11}+\left((\rho B)^{2} e^{-4 \nu} \omega^{2}-1\right) \Phi_{33}+2 e^{-4 \nu} \omega \Phi_{03}\right) \\
& +\frac{1}{B} \nabla B \cdot \nabla \nu-\nabla \nu \cdot \nabla \nu+\frac{1}{\rho} \nabla \rho \cdot \nabla \mu+\frac{1}{\rho} \nabla \rho \cdot \nabla \nu \\
& +\frac{1}{4} e^{-4 \nu}(\rho B)^{2} \nabla \omega \cdot \nabla \omega, \\
\Delta \omega= & \frac{16 \pi}{(\rho B)^{2}}\left(\Phi_{03}+(\rho B)^{2} \omega \Phi_{33}\right)-\frac{3}{B} \nabla B \cdot \nabla \omega+4 \nabla \nu \cdot \nabla \omega \\
& -\frac{2}{\rho} \nabla \rho \cdot \nabla \omega,
\end{aligned}
$$


where $\Delta u:=\rho^{-1} \partial_{\rho}\left(\rho \partial_{\rho} u\right)+\partial_{z} \partial_{z} u$ and $\nabla u=\left(\partial_{\rho} u, \partial_{z} u\right)$. The variables $\Phi_{i j}$ represent convenient combinations of the energy-momentum integrals given by

$$
\begin{aligned}
& \Phi_{00}=e^{2 \mu-2 \nu} T_{t t}, \quad \Phi_{11}=T_{\rho \rho}+T_{z z}, \\
& \Phi_{33}=(\rho B)^{-2} e^{2 \mu+2 \nu} T_{\varphi \varphi}, \quad \Phi_{03}=e^{2 \mu+2 \nu} T_{t \varphi} .
\end{aligned}
$$

For the present work we use a generalized polytropic Ansatz given by

$$
F(E, L)=A\left(E_{0}-E\right)_{+}^{k}\left(L-L_{0}\right)_{+}^{l},
$$

for an amplitude $A$, and where $(\cdot)_{+}$indicates that only the positive part is taken. Furthermore, we make the "democratic choice" $k=l=0$, meaning that all particle energies (respectively, particle angular momenta) are equally weighted. In this Ansatz the parameter $E_{0}$ specifies the maximum energy of a particle in the body, while the parameter $L_{0}$ specifies the minimum particle angular momentum. For any choice of parameters $E_{0}, L_{0}$, and a given metric, the amplitude $A$ is fixed by taking the solution to have total mass $\mathcal{M}$. In our simulations $\mathcal{M}$ is taken to be 1. The result is a two-parameter family of Ansätze parametrized by $E_{0}, L_{0}$.

The system in Eqs. (2.5)-(2.8) is solved numerically by an adaptive finite element method implemented with FEniCS [22,23], using a code built on the one described in [1]. The main addition is an adaptive mesh refinement scheme which is necessary in order to resolve the extremely dense configurations while maintaining appropriate asymptotic boundary conditions. Our mesh refinement scheme uses error indicators constructed from the jump in the normal derivative of $\nu$ across the cell boundaries and implements Dörfler marking [24] with a 35\% marking fraction. We have also implemented an Anderson $[25,26]$ acceleration scheme with variable depth (set to 4 for the extreme solutions presented in this article), which improves the convergence and stability of the fixed-point iteration. Stationary solutions presented in this paper satisfy the nonlinear system to a tolerance of $10^{-6}$. Details of the method will be presented elsewhere.

\section{B. Solution characteristics}

In any axisymmetric and stationary solution, the total mass $\mathcal{M}$ and angular momentum $\mathcal{J}$ can be computed via Komar integrals [27], for which we obtain

$$
\begin{aligned}
\mathcal{M}= & 2 \pi \int_{z=-\infty}^{\infty} \int_{\rho=0}^{\infty} B\left(\Phi_{00}+\Phi_{11}\right. \\
& \left.+\Phi_{33}\left(1-(\rho B)^{2} \omega^{2} e^{-4 \nu}\right)\right) \rho \mathrm{d} \rho \mathrm{d} z \\
\mathcal{J}= & -2 \pi \int_{z=-\infty}^{\infty} \int_{\rho=0}^{\infty} e^{-4 \nu} B\left(\Phi_{03}+\omega(\rho B)^{2} \Phi_{33}\right) \rho \mathrm{d} \rho \mathrm{d} z .
\end{aligned}
$$

When comparing our regular solutions to black hole solutions, it is useful to distinguish the cases where $|\mathcal{J}| / \mathcal{M}^{2}$ is greater or less than 1.

A quantity of interest is the binding energy (or mass defect), which specifies how much energy would be released upon forming the gravitationally bound body (cf. [28]). Below in Sec. III we give values for the fractional binding energy $E_{b}=\left(\mathcal{M}_{0}-\mathcal{M}\right) / \mathcal{M}_{0}$, which is the ratio of the binding energy to the total rest mass $\mathcal{M}_{0}$, where

$$
\begin{aligned}
\mathcal{M}_{0}= & 2 \pi \int_{z=-\infty}^{\infty} \int_{\rho=0}^{\infty} B e^{2 \mu}\left(\int_{\mathcal{P}} f(\rho, z, p)\right. \\
& \left.\times p^{0} \frac{\sqrt{-\operatorname{det} g}}{-p_{0}} \mathrm{~d} p^{1} \mathrm{~d} p^{2} \mathrm{~d} p^{3}\right) \rho \mathrm{d} \rho \mathrm{d} z .
\end{aligned}
$$

Another characterization of a solution geometry is the redshift. We characterize the redshift using the zero angular momentum observers [29] for which the redshift of a photon emitted at $(\rho, z)$ and observed at spatial infinity is given by $Z(\rho, z)=e^{-\nu(\rho, z)}-1$. For solutions approaching a black hole, the field $\nu$ (which is negative) becomes unbounded, and it is useful to instead use the rescaled quantity

$$
\bar{Z}:=\frac{Z}{1+Z}=1-e^{\nu},
$$

which in the black hole limit approaches 1 . We give values for this quantity at the peak density of the body, denoted $\bar{Z}_{p}$.

In spherical symmetry, a black hole forms if the mass $\mathcal{M}$ becomes confined within a Schwarzschild radius of $\mathcal{R}=2 \mathcal{M}$. The compactness $2 \mathcal{M} / \mathcal{R}$ is thus a useful characterization of the solution. There are no such welldefined criteria in axisymmetry. In our setting, a natural measure of the radius is the length of the axisymmetric Killing vector field which we denote $R_{\text {circ }}:=\rho B e^{-\nu}$. This quantity provides a natural length scale for the solution, in particular when restricted to the reflection plane $(z=0)$ and evaluated near the boundary of the matter. For Vlasov matter, which typically has an extended atmosphere, it is useful to take the radius at which the ratio $m / R_{\text {circ }}$ inside a cylinder of radius $\rho$ is maximum. We define the compactness parameter

$$
\Gamma:=\max _{\rho \in(0, \infty)} 2 m(\rho) / \bar{R}_{\text {circ }}(\rho),
$$

where $\bar{R}_{\text {circ }}:=\left.\left(R_{\text {circ }}\right)\right|_{z=0}$ and where

$$
\begin{aligned}
m(\rho):= & 2 \pi \int_{z=-\infty}^{\infty} \int_{\tilde{\rho}=0}^{\rho} B\left(\Phi_{00}+\Phi_{11}\right. \\
& \left.+\Phi_{33}\left(1-(\tilde{\rho} B)^{2} \omega^{2} e^{-4 \nu}\right)\right) \tilde{\rho} \mathrm{d} \tilde{\rho} \mathrm{d} z .
\end{aligned}
$$

Note that $m(\rho)=\mathcal{M}$ when $\rho$ exceeds the matter support. For the regular solutions we construct $\Gamma \in(0,1)$. 


\section{RESULTS}

\section{A. Overview}

To investigate the limiting behavior of solutions under the Ansatz Eq. (2.10), we construct sequences of stationary solutions via a path-following approach starting from an initial spherically symmetric solution with a diffuse density profile. For each sequence the $L_{0}$ parameter is fixed and the $E_{0}$ parameter is stepped down, where at each step the previous converged solution is provided as an initial guess to the solver. The behavior of various physical characteristics is investigated along the sequence and also compared with sequences of different $L_{0}$ parameters. Solution sequences for several values of $L_{0}$ were computed and in Fig. 1 we illustrate the behavior of the $E_{0}, L_{0}$ parameter space. Our studies indicate essentially two regimes, corresponding to low and high total angular momentum solutions, and one can tune between these by varying the minimum particle angular momentum $L_{0}$. Plots of various solution characteristics for three select sequences $L_{0}=0.6,0.80625,0.95$ in Fig. 2 illustrate the behavior in the different regimes.

In Fig. 1(a) the fractional binding energy $E_{b}$ is plotted against $E_{0}$ for several different $L_{0}$ values. A conjecture of Zel'dovich [28] says that steady state solutions in a

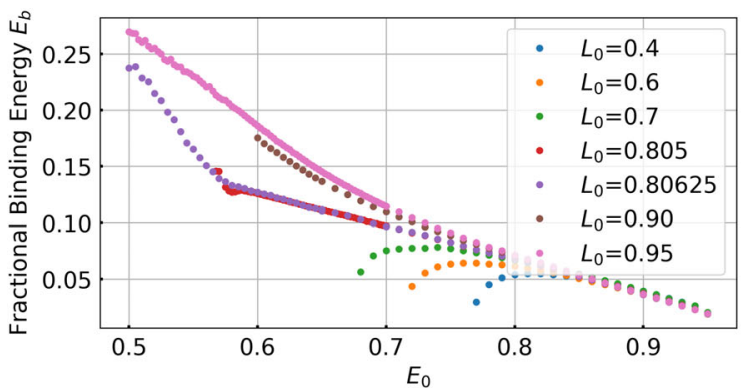

(a)

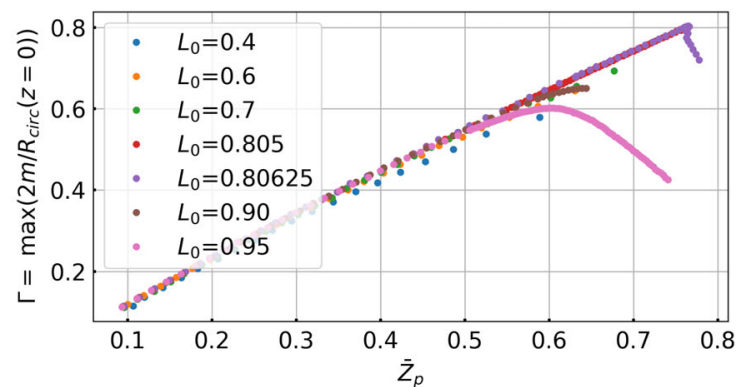

(b)

FIG. 1. Panel (a) shows the fractional binding energy $E_{b}$ for a range of solution sequences with different $L_{0}$-parameters. Panel (b) illustrates a linear relationship between the compactness $\Gamma$ and the peak redshift $\bar{Z}_{p}$ for stationary solutions along a main sequence, and the failure of this relationship for solutions tending towards the string limit.

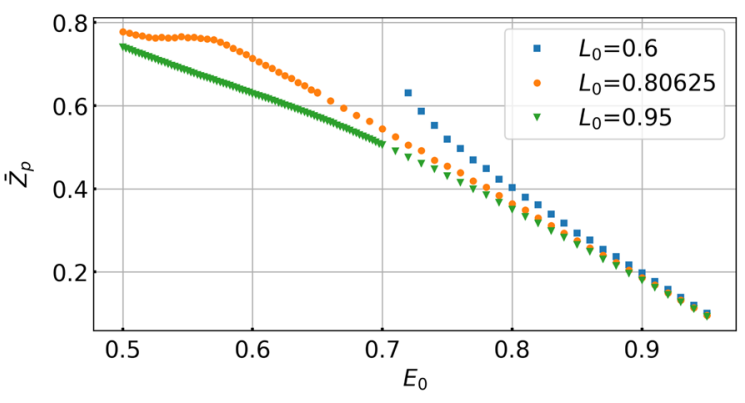

(a)

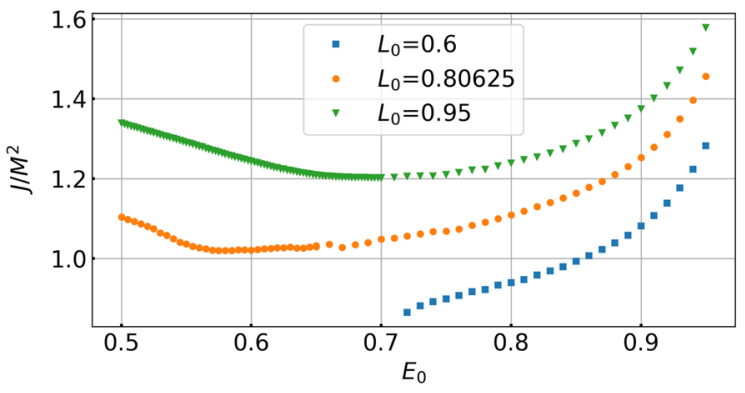

(c)

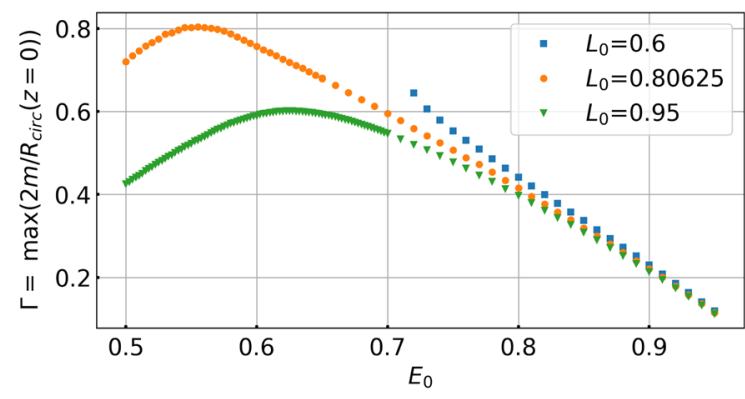

(b)

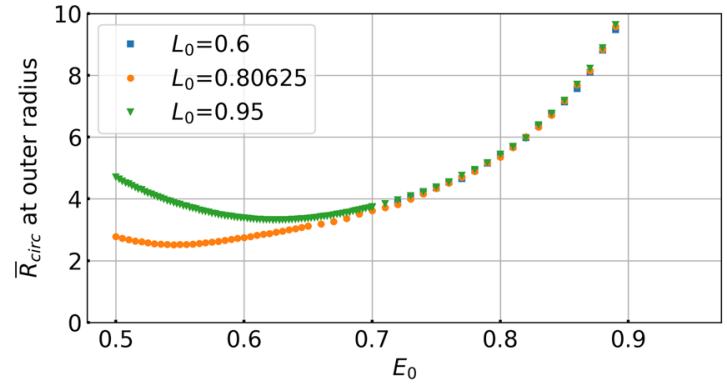

(d)

FIG. 2. Solution characteristics for three different solution sequences plotted versus $E_{0}$. Definitions of the characteristics can be found in Sec. II B. Panel (a) shows the renormalized redshift at the peak in the energy density, Eq. (2.14). Panel (b) displays the compactness parameter $\Gamma$, Eq. (2.15). In panel (c) the ratio of total angular momentum to total mass squared is plotted [cf. Eqs. (2.11) and (2.12)]. Panel (d) displays $\bar{R}_{\text {circ }}\left(\rho_{\text {outer }}\right)$; cf. the definition of $\Gamma$. 
parametrized family, which are past the maximum in the binding energy, are dynamically unstable. For solution sequences in the low-angular momentum regime [e.g., $L_{0}=0.4,0.6,0.7$ in Fig. 1(a)], such a maximum is observed, and shortly after the maximum the residual in our numerical fixed-point iteration grows and the numerics break down. Furthermore, such solutions become subcritical in that $|\mathcal{J}| / \mathcal{M}^{2}<1$ [cf. e.g., the $L_{0}=0.6$ sequence in Fig. 2(c)]. It is our hypothesis that solutions sufficiently far past the maximum in the binding energy become unstable to gravitational collapse via modes accessible in axisymmetry. Further work to investigate instability in the axisymmetric setting numerically is in progress [30].

While solution sequences in the low total angular momentum regime eventually terminate after a maximum in the binding energy, this maximum occurs at lower $E_{0}$ values for sequences with greater $L_{0}$ value. For sufficiently large $L_{0}$ values, $E_{b}$ appears to be monotonically increasing with decreasing $E_{0}$, and $|\mathcal{J}| / \mathcal{M}^{2}$ is always greater than 1 . Due to their supercritical total angular momentum, such solutions cannot collapse to a black hole in axisymmetry where gravitational waves carry no angular momentum. A natural question to ask is which $L_{0}$ parameter separates the low and high total angular momentum regimes. Via a bisection search we have determined this critical value to be $L_{0}=0.805625$ to within \pm 0.000625 . Referring to Fig. 1(a), the $L_{0}=0.805$ sequence (red in color version) was determined to be subcritical in that a peak in the binding energy appeared, whereas for the $L_{0}=0.80625$ sequence (purple in color version) no such peak appears. Since the numerical computation of each sequence is extremely computationally expensive, we halted the bisection search after $L_{0}=0.80625$. In Sec. III B we present numerical evidence that the critical solution sequence approaches an extremal Kerr black hole solution.

An interesting property of the solution sequences is illustrated in Fig. 1(b). Here it is shown that solutions can be classified as either lying on or off a main sequence. Solutions on the main sequence correspond to those with a major axis (as measured by $R_{\text {circ }}$ ) that shrinks with increasing redshift, resulting in an increasing compactness parameter $\Gamma$. At high redshift, such solutions appear to be approaching a black hole limit, as argued in Sec. III B. However, solution sequences with a sufficiently large angular momentum [such as the $L_{0}=0.80625$ and $L_{0}=$ 0.95 solution sequences shown in Fig. 1(b)] eventually leave the main sequence and tend towards a different limit, along which $R_{\text {circ }}$ is increasing. ${ }^{1}$ Properties of solutions at the extreme of this portion of the sequence are discussed in Sec. III C. In particular, the major axis grows, while the

\footnotetext{
${ }^{1}$ The $L_{0}=0.9$ sequence can also be seen to leave the main sequence. However, this sequence was not pursued to lower $E_{0}$ values, so the departure is not as dramatic.
}

minor axis of the configuration continues to decrease, resulting in near-Dirac-type matter distributions.

It is also interesting to observe that for solution sequences with ergoregions, those ergoregions first appear near $E_{0}=0.66$, independently of the $L_{0}$ parameter. Density profiles and accompanying ergoregions, if present, are shown in Figs. 3 and 4 for a selection of solutions on the $L_{0}=0.80625$ sequence.

\section{B. A quasistationary transition to an extreme Kerr black hole}

Our bisection search indicates that the $L_{0}=0.80625$ solution sequence is near critical and remains on the stable side of the binding energy curve as $|\mathcal{J}| / \mathcal{M}^{2} \rightarrow 1$ from above. In this section we show that as $E_{0} \rightarrow 0.58$, solutions in this sequence appear to come close to an extremal Kerr black hole solution, and we demonstrate that a quasistationary transition to the extremal Kerr black hole is plausible for a critical solution sequence in this family. After $E_{0}=0.58$, the solutions change course and tend towards the string limit discussed in Sec. IIIC. We emphasize at this point that prior to this study nothing was known about black hole limits of families of stationary axisymmetric Einstein-Vlasov bodies, and there is no a priori requirement known to the authors that any limiting black hole to a family of Einstein-Vlasov solutions be in the Kerr family or be an extremal black hole.

With the coordinates and metric parametrization used here, the extreme Kerr solution of mass $\mathcal{M}$ has a metric of the form Eq. (2.1) with [31,32]

$$
\begin{aligned}
\nu_{E K} & =\frac{1}{2} \ln \left(\frac{r^{2}(\mathcal{M}+r)^{2}+\mathcal{M}^{2} z^{2}}{\left(\mathcal{M}^{2}+(\mathcal{M}+r)^{2}\right)^{2}-\mathcal{M}^{2} \rho^{2}}\right), \\
B_{E K} & =1, \\
\mu_{E K} & =\frac{1}{2} \ln \left(r^{-2}(\mathcal{M}+r)^{2}+\mathcal{M}^{2} z^{2} r^{-4}\right), \\
\omega_{E K} & =\frac{2 \mathcal{M}^{2}(\mathcal{M}+r)}{\left(\mathcal{M}^{2}+(\mathcal{M}+r)^{2}\right)^{2}-\mathcal{M}^{2} \rho^{2}},
\end{aligned}
$$

where $r^{2}=\rho^{2}+z^{2}$. In these coordinates the horizon is at $\rho=z=0$, and the exterior vacuum spacetime is coordinatized on the domain $\rho \in(0, \infty), z \in(-\infty, \infty)$.

Using the expressions (3.1) and (3.2), it can be shown that $\bar{R}_{\text {circ }}(\rho)$ (cf. Sec. II B) is an increasing function of $\rho$ and that $\lim _{\rho \rightarrow 0} \bar{R}_{\text {circ }}(\rho)=2 \mathcal{M}$. Thus, for an extremal Kerr black hole, both the compactness parameter $\Gamma$ and the normalized peak redshift $\bar{Z}_{p}$ are unity. In Figs. 2(a), 2(b), and 1(b), it is shown that for the near-critical solution sequence, these characteristics increase towards unity as $E_{0} \rightarrow 0.58$.

The hoop conjecture [33] says that a body collapses to a black hole if and only if the radius of the body is less than or equal to its Schwarzschild radius, $2 \mathcal{M}$. If we use $\bar{R}_{\text {circ }}$ 


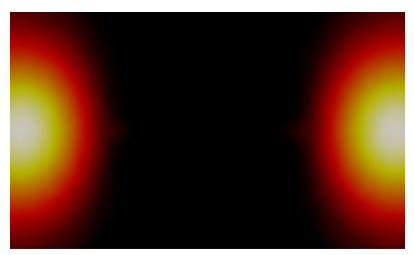

(a) $E_{0}=0.8$

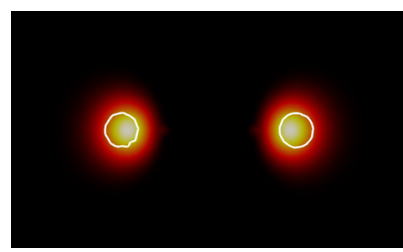

(d) $E_{0}=0.65$

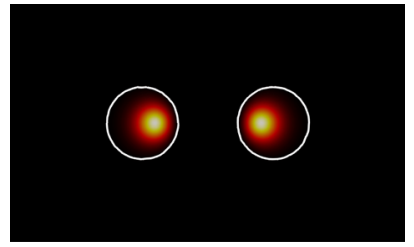

(g) $E_{0}=0.58$

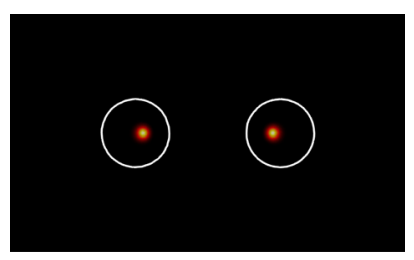

(j) $E_{0}=0.52$

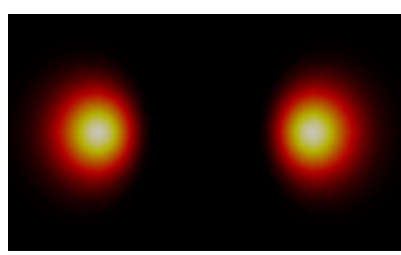

(b) $E_{0}=0.7$

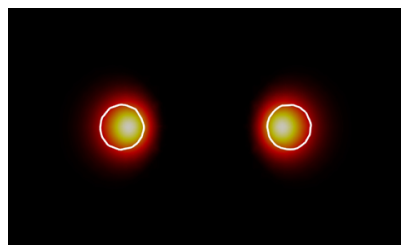

(e) $E_{0}=0.64$

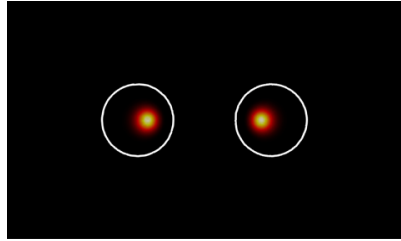

(h) $E_{0}=0.54$

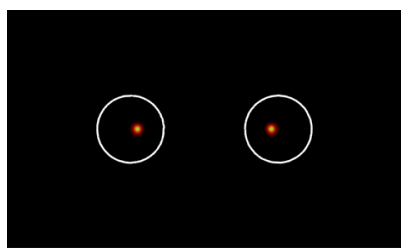

(k) $E_{0}=0.51$

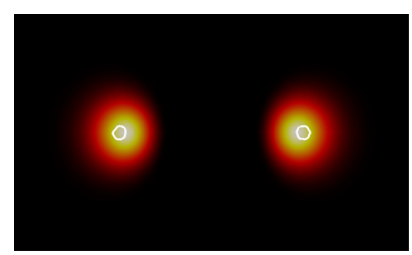

(c) $E_{0}=0.66$

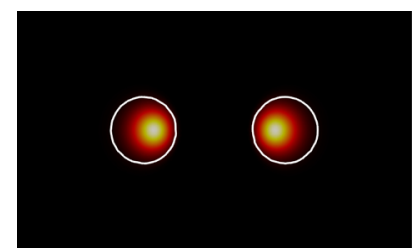

(f) $E_{0}=0.6$

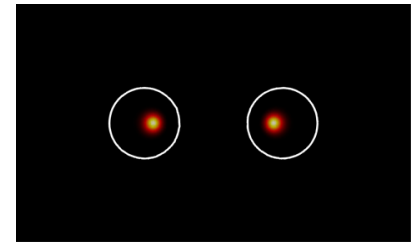

(i) $E_{0}=0.53$

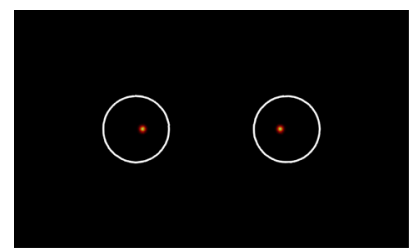

(1) $E_{0}=0.5$

FIG. 3. Energy density (heat map) and ergoregion (boundary shown by white trace) for a selection of solutions on the $L_{0}=0.80625$ solution sequence. The heat map is rescaled for each stationary solution.

measured at the outer radius of support as a measure of the body radius, the hoop conjecture implies that a black hole will form when this radius approaches 2 (noting that in our simulations $\mathcal{M}$ is set to 1 ). This quantity is plotted in
Fig. 2(d), where it is observed that for the near-critical sequence, $\bar{R}_{\text {circ }}$ reaches a minimum of $\sim 2.5$ as $E_{0} \rightarrow 0.58$. We note that this value is also consistent with the maximum compactness $\sim 0.8$.

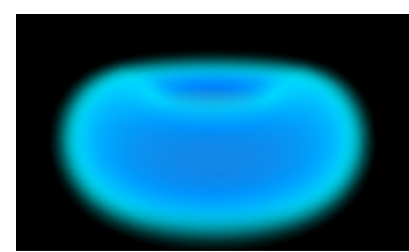

(a) $E_{0}=0.7$

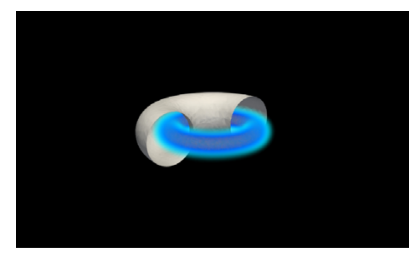

(d) $E_{0}=0.54$

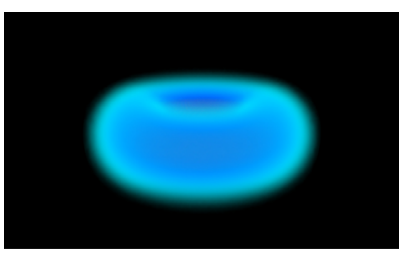

(b) $E_{0}=0.65$

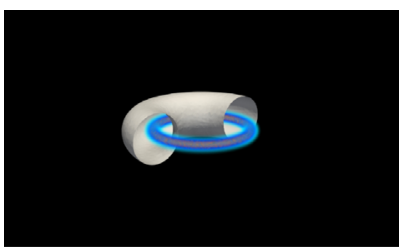

(e) $E_{0}=0.52$

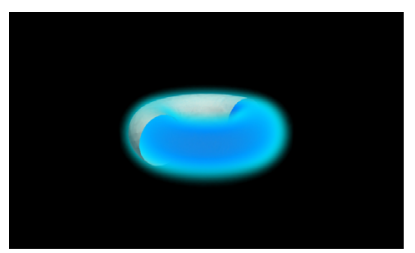

(c) $E_{0}=0.6$

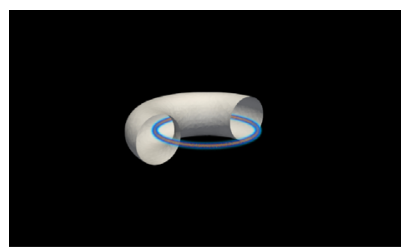

(f) $E_{0}=0.5$

FIG. 4. Energy density (blue) and ergoregion (surface shown in white) for a selection of solutions on the $L_{0}=0.80625$ solution sequence. The color map is rescaled for each stationary solution. 


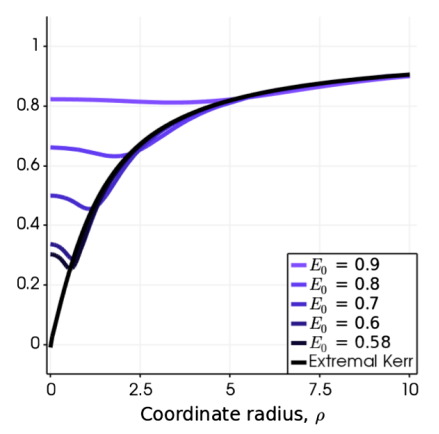

(a)

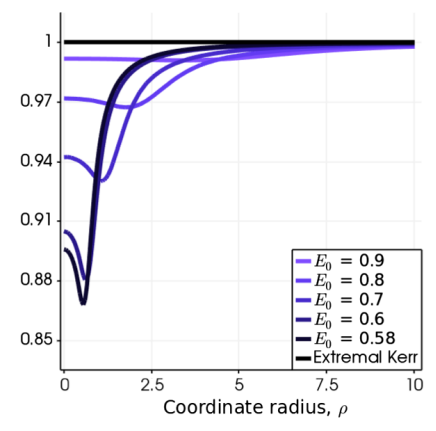

(b)

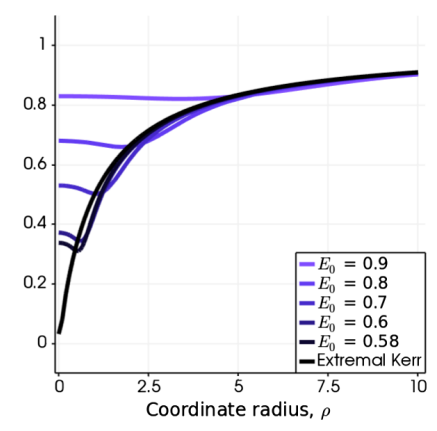

(c)

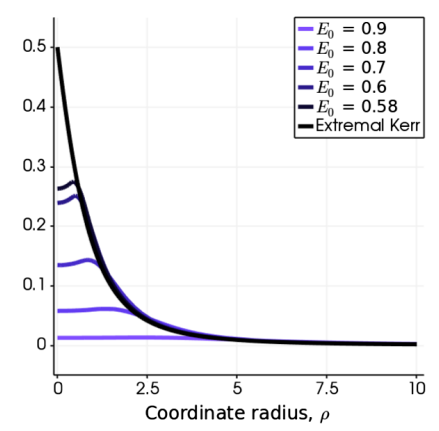

(d)

FIG. 5. Cross sections in the reflection plane $(z=0)$ of the metric fields for a selection of solutions on the $L_{0}=0.80625$ solution sequence approaching the black hole limit, and comparison to the extreme Kerr solution (black). Panel (a) $\operatorname{shows} \exp (\nu)$, (b) $B$, (c) $\exp (-\mu)$, and in (d) the $\omega$ field.

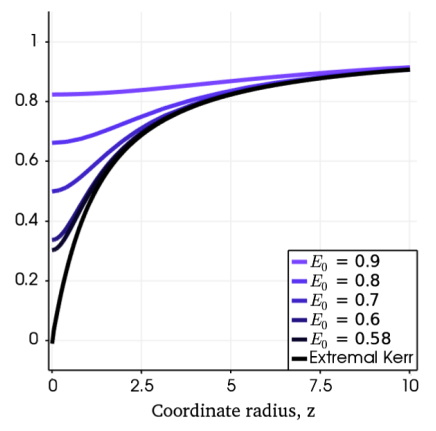

(a)

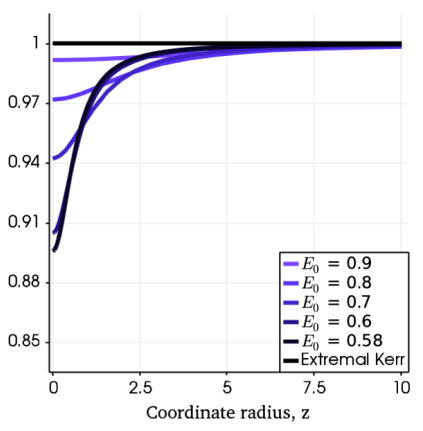

(b)

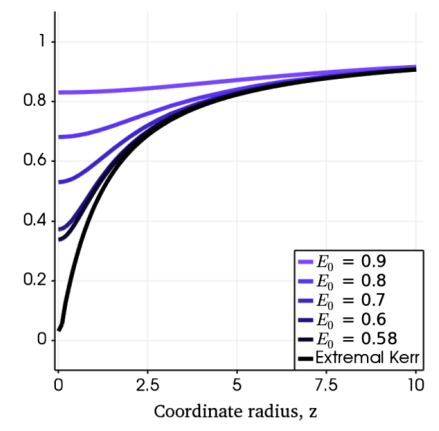

(c)

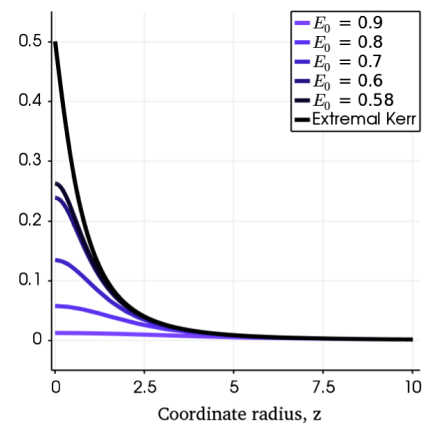

(d)

FIG. 6. Cross sections on the axis $(\rho=0)$ of the metric fields for a selection of solutions on the $L_{0}=0.80625$ solution sequence approaching the black hole limit, and comparison to the extreme Kerr solution (black). Panel (a) $\operatorname{shows} \exp (\nu),(\mathrm{b}) B$, (c) $\exp (-\mu)$, and in (d) the $\omega$ field.

We have also investigated the metric fields for the near-critical sequence. In Figs. 5 and 6, the limiting behavior of the metric fields is shown for traces in the reflection plane and on the axis, respectively, and compared with that of the extremal Kerr black hole solution. The $r \rightarrow 0$ limit, corresponding to the black hole horizon, implies [cf. Eqs. (3.1)-(3.4)] $\nu_{E K} \rightarrow-\infty, \mu_{E K} \rightarrow \infty$, and $\omega_{E K} \rightarrow 1 /(2 \mathcal{M})$. Thus, in order to compare quantities which are regular on the horizon, we plot $e^{\nu}$ [panel (a)], $B$ [panel (b)], $e^{-\mu}$ [panel (c)], and $\omega$ [panel (d)]. The plots show that the metric fields of solutions on the main sequence before $E_{0} \approx 0.58$ exhibit a steady approach to those of the extremal Kerr solution. After $E_{0} \approx 0.58$, the fields cease this approach and appear to converge to a different solution at larger radius, as illustrated in Figs. 7 and 8 .

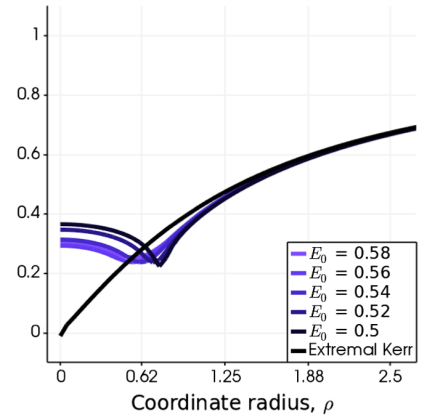

(a)

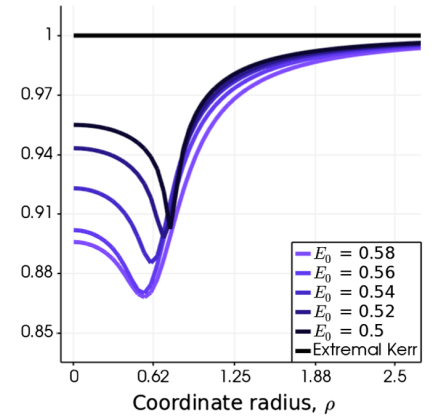

(b)

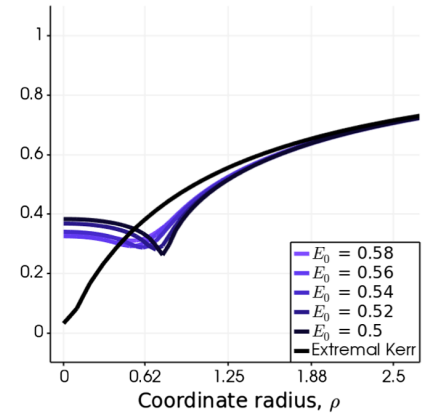

(c)

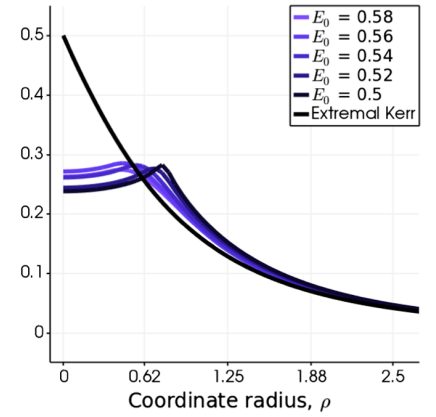

(d)

FIG. 7. Cross sections in the reflection plane $(z=0)$ of the metric fields for a selection of solutions on the $L_{0}=0.80625$ solution sequence approaching the thin-ring limit, and comparison to the extreme Kerr solution (black). Panel (a) $\operatorname{shows~} \exp (\nu)$, (b) $B$, (c) $\exp (-\mu)$, and in (d) the $\omega$ field. 


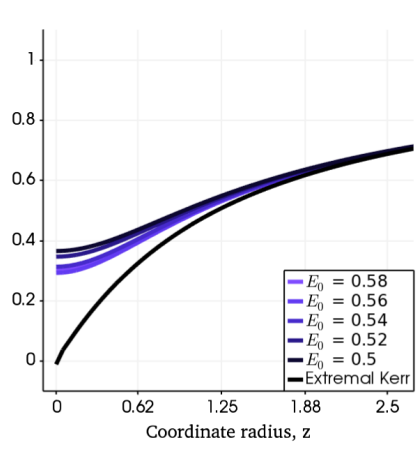

(a)

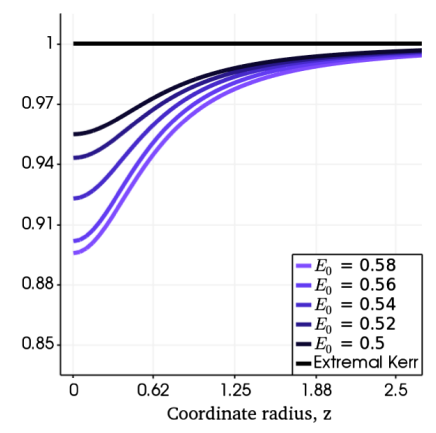

(b)

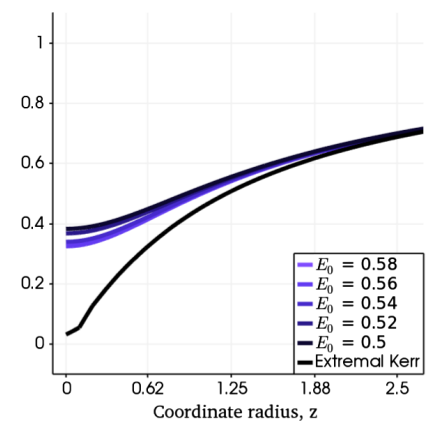

(c)

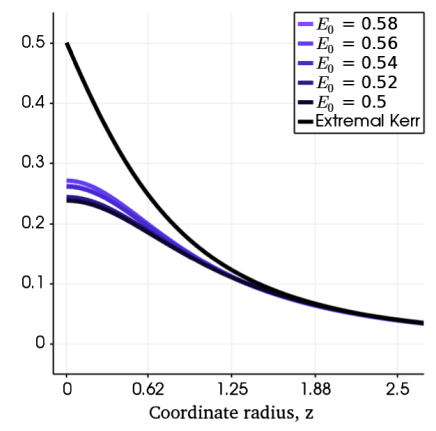

(d)

FIG. 8. Cross sections on the axis $(\rho=0)$ of the metric fields for a selection of solutions on the $L_{0}=0.80625$ solution sequence approaching the thin-ring limit, and comparison to the extreme Kerr solution (black). Panel (a) shows $\exp (\nu),(\mathrm{b}) B$, (c) $\exp (-\mu)$, and in (d) the $\omega$ field.

The fact that the extremal Kerr angular momentum to mass ratio, normalized redshift, compactness, and metric fields are all approached by the near-critical solution sequence $L_{0}=0.80625$ as $E_{0}$ approaches 0.58 suggests that solutions on this sequence near $E_{0}=0.58$ are close to the extremal Kerr solution. While our study does not rule out a Buchdahl-type gap in this setting or the existence of a quasistationary transition to more general rotating black holes, these results along with the results of Meinel [7,8] and Ansorg et al. [9-11], as well as results in the charged spherically symmetric Einstein-Vlasov setting [5], suggest the existence of a critical $L_{0}$-parameter for which the limiting member is an extremal Kerr black hole. Our study suggests that this limit is reached at a positive $E_{0}$ value.

\section{Thin-ring limits of toroidal bodies}

Families of solutions in the supercritical regime, $|\mathcal{J}| / \mathcal{M}^{2}>1$, eventually tend towards a limit which is distinct from the black hole limit. Due to the thin-ring-like nature of the energy density for extreme members of these solution sequences, we refer to this as the "thin-ring limit." Such sequences are illustrated in Fig. 2 by the $L_{0}=0.95$ solution sequence. The near-critical $L_{0}=0.80625$ solution sequence also tends towards a thin-ring limit late in the solution sequence. In fact, for larger $L_{0}$-parametrized sequences, the thin-ring limit is approached increasingly earlier in the sequence, that is, at larger $E_{0}$ values.

After branching off from the main sequence [cf. Fig. 1(b)] an $E_{0}$-parametrized sequence of solutions becomes increasingly thin and the radius of the peak density increases slightly, as can be seen in Fig. 3(h)-3(l). Figures 7 and 8 also show that along the sequence the fields move away from the black hole limit and apparently converge to a distinct limiting configuration. The geometry of extreme solutions in this limit has a near-field regime which is locally rotationally symmetric about the matter ring as illustrated by the contour plot in Fig. 9. This regime is small compared to the ring radius. Far away, the fields are, as dictated by the boundary conditions, asymptotically flat. Additionally, a computation of the Kretschmann scalar indicates that it vanishes a distance of order the ring radius from the ring.

The geometry of these thin-ring solutions suggests a comparison with circular cosmic string models [12-14]. The most relevant feature of these spacetimes is the deficit angle that characterizes a conical region near the string. Circular cosmic strings can be thought of as having two regimes, similar to the thin-ring solutions shown in Fig. 9: a near-field regime in which the string appears like an infinitely long and straight cosmic string, and a far-field regime in which the gravitational fields are asymptotically flat, reflecting the compactly supported matter distribution. The near-field regime is small compared to the major toroidal radius, and the spacetime is rotationally symmetric about the matter and conical. However, this rotational symmetry is broken on distances comparable to that of the toroidal major axis and on such scales the deficit angle and conical geometry are not well defined. Thus, whereas the deficit angle for a straight cosmic string approaches a constant well-defined value at spatial infinity, for a circular cosmic string, the deficit angle is only well-defined in the near-field rotationally symmetric region. Below, we investigate the existence of a well-defined deficit angle, and a near-field conical region in both toroidal coordinates

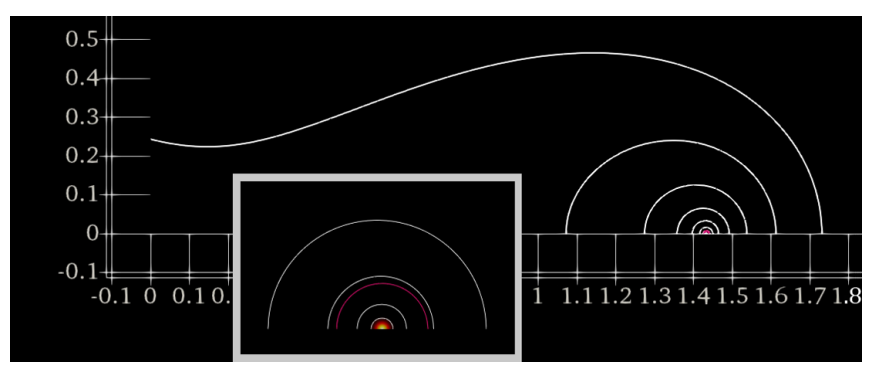

FIG. 9. Near-circular contours of the $\mu$ field in the region near the matter for the $L_{0}=0.95, E_{0}=0.5$ solution. Inset: A close-up view of the matter density with a $\sigma=6$ contour of the toroidal radius is included for reference (magenta curve). Axes ticks are in units of $\rho / \mathcal{M}$ (horizontal) and $z / \mathcal{M}$ (vertical). 


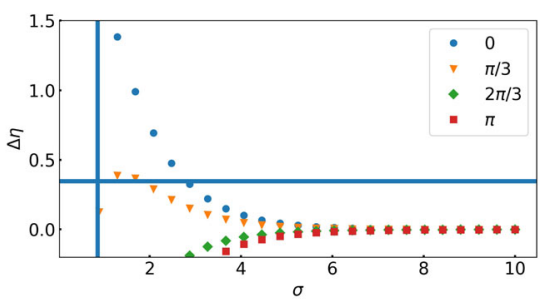

(a) $E_{0}=0.9$

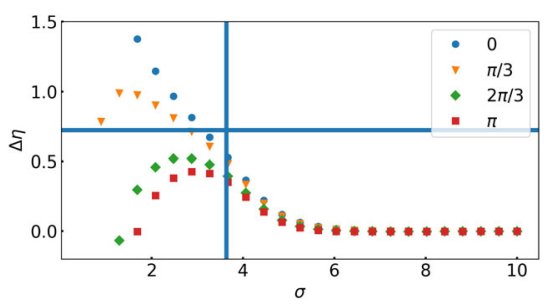

(d) $E_{0}=0.58$

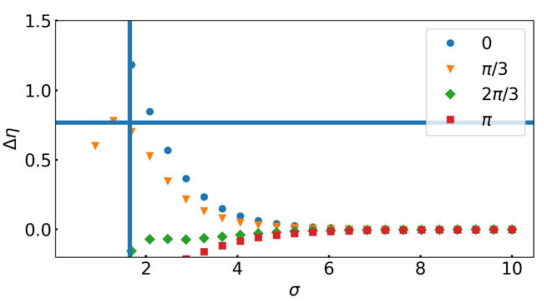

(b) $E_{0}=0.7$

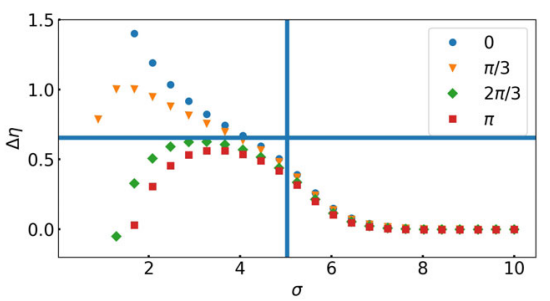

(e) $E_{0}=0.54$

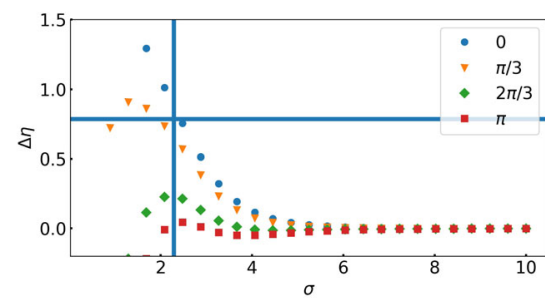

(c) $E_{0}=0.64$

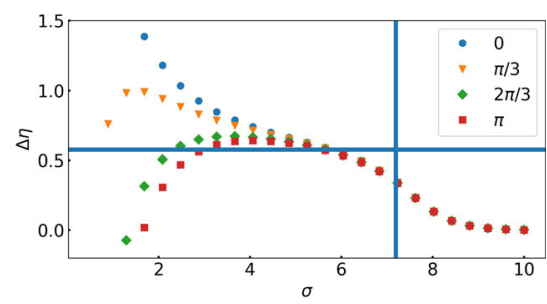

(f) $E_{0}=0.5$

FIG. 10. Deficit angle as computed with Eq. (3.5) versus $\sigma_{0}$ for $\psi_{0}=(0, \pi / 3,2 \pi / 3, \pi)$ for a selection of solutions on the $L_{0}=0.95$ solution sequence. In each panel the vertical line indicates the support of the matter computed by Eq. (3.6), and the horizontal line is an estimate of the deficit angle based on linearized theory for Dirac sources-see Eq. (3.10) and also Fig. 12(a). The ring that forms the center of the toroidal matter distribution is located at $\sigma \rightarrow \infty$.

(cf. Fig. 10) and in local polar coordinates (cf. Fig. 11). In both cases the coordinates are centered on the peak matter density.

To measure the deficit angle and facilitate comparison with previous studies on circular cosmic strings in the literature [12-14], we write the relevant part of our metric in toroidal coordinates $(\sigma, \psi) \in(0, \infty) \times[-\pi, \pi)$ defined by

$$
\rho=a N^{-2} \sinh (\sigma), \quad z=a N^{-2} \sin (\psi),
$$

where $\tilde{\mu}(\sigma, \psi)=(\mu \circ y)(\sigma, \psi)$ and $y$ is the coordinate map $(\sigma, \psi) \mapsto(\rho, z)$. The deficit angle, $\Delta \eta$, can be expressed in terms of the ratio of the proper $\psi$-arclength to proper $\sigma$-radius:

$\left.\Delta \eta\right|_{\sigma=\sigma_{0}, \psi=\psi_{0}}=2 \pi-\frac{\left.\int_{-\pi}^{\pi}\left(a N^{-2}(\sigma, \psi) e^{\tilde{\mu}(\sigma, \psi)}\right)\right|_{\sigma=\sigma_{0}} \mathrm{~d} \psi}{\left.\int_{\sigma_{0}}^{\infty}\left(a N^{-2}(\sigma, \psi) e^{\tilde{\mu}(\sigma, \psi)}\right)\right|_{\psi=\psi_{0}} \mathrm{~d} \sigma}$.

where

$$
N^{2}=\cosh (\sigma)-\cos (\psi)
$$

and $a=\rho_{\text {peak }}$ is the radial coordinate of the peak density. In these coordinates the peak in the density is obtained by the limit $\sigma \rightarrow \infty$. The metric in the meridional plane takes the form

$$
e^{2 \tilde{\mu}} a^{2} N^{-4}\left(\mathrm{~d} \sigma^{2}+\mathrm{d} \psi^{2}\right),
$$

This quantity is based at $\left(\sigma_{0}, \psi_{0}\right)$ in the sense that one must choose values at which to evaluate the integrals. If there is an open set of coordinate values $\left(\sigma_{0}, \psi_{0}\right)$ on which $\left.\Delta \eta\right|_{\sigma=\sigma_{0}, \psi=\psi_{0}}$ is constant, then the geometry is conical with deficit angle $\Delta \eta$.

The results of this calculation for a selection of solutions in the $L_{0}=0.95$ solution sequence are shown in Fig. 10, where in each panel we plot $\Delta \eta$ versus $\sigma_{0}$ for $\psi_{0}=(0, \pi / 3$, $2 \pi / 3, \pi)$, corresponding to the different color traces.

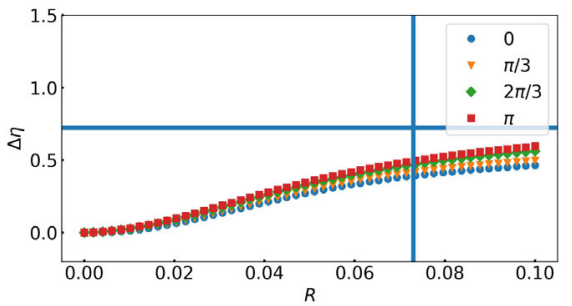

(a) $E_{0}=0.58$

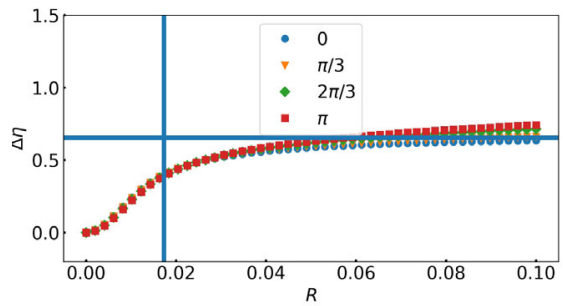

(b) $E_{0}=0.54$

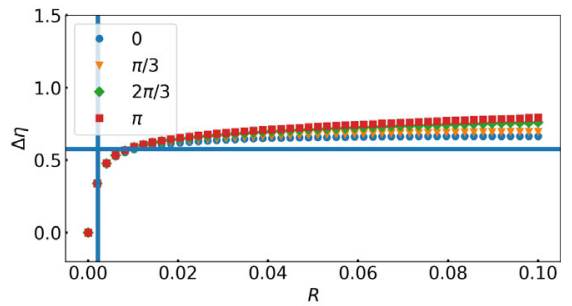

(c) $E_{0}=0.5$

FIG. 11. Deficit angle versus the local polar coordinate $R$ for $\theta=(0, \pi / 3,2 \pi / 3, \pi)$ for a selection of solutions on the $L_{0}=0.95$ solution sequence. In each panel the vertical line indicates the support of the matter computed by Eq. (3.6), and the horizontal line is an estimate of the deficit angle based on linearized theory for Dirac sources-see Eq. (3.10) and also Fig. 12(a). 
The vertical line in each panel is an estimate of the boundary of the matter $\sigma_{\text {supp }}$, which is obtained by

$\sigma_{\text {supp }}:=\min _{\tilde{\rho} \in\left\{\rho_{\text {inner }}, \rho_{\text {outer }}\right\}}\left\{\log \left(\left(\rho_{\text {peak }}+\tilde{\rho}\right) /\left(\rho_{\text {peak }}-\tilde{\rho}\right)\right)\right\}$.

Recall that larger $\sigma$ corresponds to a distance closer to the peak matter density. For reference, the $\sigma=6$ contour is included (magenta line) in the contour plot in Fig. 9. The plots in Fig. 10 have been cut off at $\sigma=10$ in order to show more detail at low $\sigma$. However, $\Delta \eta$ also remains zero (inside the matter distribution) to $\sigma$ as large as our numerical resolution allows. The horizontal line in each plot is discussed below.

Inspection of Fig. 10 shows that for solutions on the main sequence (for the $L_{0}=0.95$ solution sequence, this corresponds to $\left.E_{0}>0.64\right)$ the deficit angle is zero where it is defined and becomes undefined even within the support of the matter due to a lack of symmetry for such solutions. The transition between the near- and far-field regimes discussed above is seen in Fig. 10 by the failure of the different $\psi_{0}$ traces to agree. For more extreme members of the thin-ring sequence, $\Delta \eta$ becomes constant in $\psi_{0}$ outside of the support of the matter and takes a nonzero value. While $\Delta \eta$ is not simultaneously constant in $\psi_{0}$ and $\sigma_{0}$ for any of the solutions we compute, one notes that late in the sequence [note in particular Fig. 10(f)] a region begins to form where $\Delta \eta$ appears to approach a constant in both $\psi_{0}$ and $\sigma_{0}$, suggestive of a locally conical region.

While toroidal coordinates are useful global coordinates, they can be counterintuitive since the center of the toroidal matter density is located at $\sigma \rightarrow \infty$. Local polar coordinates $(R, \theta)$ centered at the peak density defined by $\rho=\rho_{\text {peak }}+R \cos (\theta), z=R \sin (\theta)$, are suitable for investigating the near-ring geometry. The deficit angle can be computed in a similar way as in Eq. (3.5), and this is plotted in Fig. 11 for a selection of solutions. These plots further support the picture of a locally conical region sufficiently close to the matter support. In particular, the most extreme solution we compute along this sequence, shown in Fig. 11(c), shows a near-step-function-like graph characteristic of a conical region.

The trend in the computation of $\Delta \eta$ just illustrated suggests to the authors that limiting members of such sequences exhibit a local region about a near-Dirac-type matter distribution in which $\Delta \eta$ is constant and the geometry is conical. We stress at this point, however, that since we can only investigate the trend of $\Delta \eta$ along solution sequences, and not the geometry of the limiting members of these sequences, that the conical nature of the geometry for such limiting members is far from certain. Nonetheless, inspired by this trend, we find it of interest to compare the extreme members of high angular momentum solution sequences to circular cosmic string models with a prescribed Dirac source. As pointed out by Garfinkle and coauthors [34-36], in general relativity the gravitational field should be found by solving the coupled Einsteinmatter system for an appropriate matter model rather than through prescribing a fixed energy-momentum tensor. They find, however, in the case of certain Einstein-scalar-gauge field models for infinitely long straight cosmic strings that the deficit angle can be approximated by the result obtained through a prescribed Dirac energy-momentum and linearized gravity originally derived by Vilenkin [37]. In the case of circular cosmic strings Hughes et al. [13] and McManis and Vandyck [14] have adapted the approach of Vilenkin. They specify a Dirac source at $\rho=a, z=0$ with a linear energy density $u=-T_{t}{ }^{t}$ and linear azimuthal pressure $k=T_{\varphi}{ }^{\varphi}$. Using the linearized Einstein equations it is found - both without [13] and with [14] rotation-that the deficit angle $\Delta \eta$ can be approximated by $4 \pi(u-k)$ (setting $G=1$ ).

Since the solutions presented in this paper have regular (nondistributional) energy momentum, in order to compare with the $[13,14]$ results, we compute the corresponding quantities $u$ and $k$ by integrating the components of the energy-momentum tensor over the meridional plane. In addition, the solutions of $[13,14]$ are supported by an external radial (meaning $\rho$ ) pressure, while the solutions presented here have intrinsic positive pressure in the meridional plane. We find that adding this meridional pressure in our deficit angle approximation improves agreement with the deficit angle computed via Eq. (3.5). Using the metric Eq. (2.1) and the integration measure $e^{2 \mu} \mathrm{d} \rho \mathrm{d} z$ on the meridional plane, we find

$$
\begin{aligned}
u= & \int_{\mathbb{R}^{2}} e^{2 \mu-2 \nu}\left(T_{t t}+\omega T_{t \varphi}\right) \mathrm{d} \rho \mathrm{d} z \\
k= & \int_{\mathbb{R}^{2}}\left((\rho B)^{-2} e^{2 \mu+2 \nu}\left(1-(\rho B)^{2} \omega^{2} e^{-4 \nu}\right) T_{\varphi \varphi}\right. \\
& \left.-\omega e^{2 \mu-2 \nu} T_{t \varphi}\right) \mathrm{d} \rho \mathrm{d} z \\
m= & \int_{\mathbb{R}^{2}}\left(T_{\rho \rho}+T_{z z}\right) \mathrm{d} \rho \mathrm{d} z .
\end{aligned}
$$

Combining and writing in terms of the $\Phi$-quantities [cf. Eq. (2.9)], we obtain

$$
\begin{aligned}
4 \pi(u+m-k)= & 4 \pi \int_{\mathbb{R}^{2}}\left(\Phi_{00}+\Phi_{11}+2 \omega e^{-4 \nu} \Phi_{03}\right. \\
& \left.-\left(1-(\rho B)^{2} \omega^{2} e^{-4 \nu}\right) \Phi_{33}\right) \rho \mathrm{d} \rho \mathrm{d} z .
\end{aligned}
$$

Note that there is a balancing of terms. The $\Phi_{00}$ and $\Phi_{11}$ terms are always positive, the $\Phi_{03}$ term is negative and grows with increasing $\omega$, while the $\Phi_{33}$ term is negative outside of an ergoregion, but becomes positive within an ergoregion. The net result is the behavior shown in Fig. 12(a) and by the horizontal line in each plot of Figs. 10 and 11. It is interesting that extreme members along the string sequence have a deficit angle that grows 


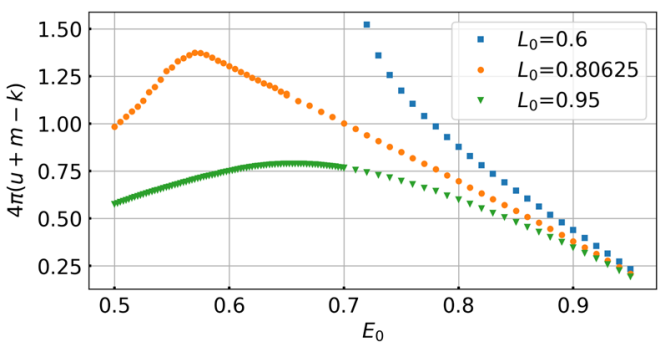

(a)

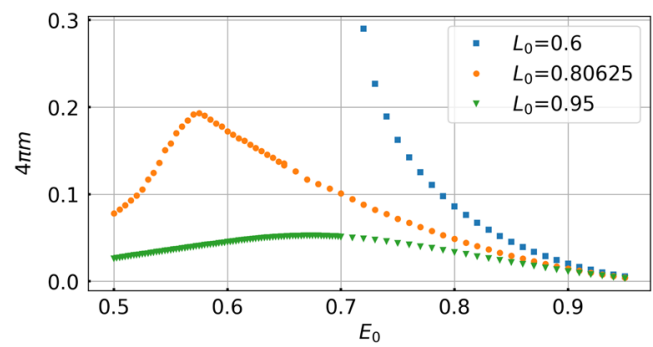

(b)

FIG. 12. (a) The estimated deficit angle Eq. (3.10). (b) The contribution from the meridional pressure, given by $4 \pi m$ for $m$ as in Eq. (3.9).

to approximately this value outside the support of the matter and before transitioning to the far-field regime. In addition, the pressure in the meridional plane decreases along the string sequence [cf. Fig. 12(b)], so that in the limit $4 \pi(u-k)$ is likely a good approximation to the deficit angle. We note that similar behavior, including agreement of the deficit angle with the result of Eq. (3.10), occurs for the extreme members of the near-critical $L_{0}=$ 0.080625 solution sequence.

\section{DISCUSSION AND CONCLUSIONS}

We have investigated a two-parameter family of toroidal stationary solutions to the Einstein-Vlasov system, and find evidence for two interesting physical limits. In Sec. III B we show that the Einstein-Vlasov system likely admits a quasistationary approach to an extremal Kerr black hole. This result is similar to that identified in axially symmetric spacetimes with uniformly rotating fluids [7-11]. We also show in Sec. III C that high angular momentum solutions exhibit a distinct solution path, along which the peak in the density moves to larger radii and the spatial density becomes supported on a thin ring. The geometry of solutions along such sequences is investigated, and the gravitational field is observed to become increasingly rotationally symmetric about the matter ring in the nearfield regime. While the geometry of the limiting solutions along such sequences is not certain, we find the trend suggestive of a locally conical region forming around a near-Dirac-type matter distribution. We have compared extreme members of these sequences, which are fully self-consistent solutions of the Einstein-Vlasov system, with models for circular cosmic strings with prescribed Dirac sources, and note that the geometry and estimated deficit angle of the conical region is consistent with the results of [12-14].

It should be noted that the solutions presented in this paper have intrinsic positive pressure. This can be compared with solutions based on Dirac sources $[13,14]$, which require negative azimuthal pressure in order to preserve Lorentz invariance, and also with the fully self-consistent solutions with gauge field sources (e.g., [35]) for which the longitudinal pressure is negative.

To what extent can the limits of these solution sequences be pushed further? In the thin-ring limit, our computation is eventually resolution limited even with mesh refinement. The matter becomes unresolved and lost by the code, resulting in a "zero mass distribution" error and code breakdown. It is likely that the limiting solution along such sequences has a Dirac distribution with radius and terminating $E_{0}$ value depending on the $L_{0}$ value. We believe that our solution sequences could be pushed further towards this limit by increasing resolution. While it is unlikely that such relativistic solutions are stable in full general relativity (without symmetry), their large angular momentum makes them stable against black hole collapse when restricted to axisymmetry. To push the low angular momentum sequences further on the unstable side of the binding energy curve and also get closer to the black hole limit, there is some evidence that a different numerical method may be required.

While stability in the axisymmetric Einstein-Vlasov system is largely wide open, in spherical symmetry the numerical study [38] provides a characterization of stable and unstable static solutions. In this setting, we have compared a fixed-point algorithm similar to the one employed in this paper with existing ODE-based methods. While the ODE methods are able to construct unstable solutions, the fixed-point algorithm only converges, roughly, in the case of stable solutions. We note that all of the nearextremal solutions (either in the black hole limit or in the string limit) contain ergoregions. Compact but non-blackhole objects containing ergoregions are considered to be unstable and short lived [39,40]. Lastly, numerical experiments to investigate the stability of axisymmetric stationary Einstein-Vlasov solutions are under way [30].

Finally we briefly comment on the potential physical relevance of these solutions. We have solved the equations with particle mass $m_{p}$ and total mass $\mathcal{M}$ both equal to 1 . To get back a solution in physical units, one may choose a value for $\mathcal{M}$ and rescale, for instance, the radius $r \rightarrow \mathcal{M r}$ and total angular momentum by $\mathcal{J} \rightarrow \mathcal{M}^{2} \mathcal{J}$ (see, e.g., [19]). 
The parameter $L_{0}$ has units of particle angular momentum, equivalent to $m_{p} \mathcal{M}$. There are no bounds on the length scale associated to the solutions presented in this paper, and thus under such a rescaling the solutions may represent objects from very small to astrophysical scales.

\section{ACKNOWLEDGMENTS}

The authors thank Reinhard Meinel for useful comments during completion of this work. E. A. is supported by the Knut and Alice Wallenberg Foundation.
[1] E. Ames, H. Andréasson, and A. Logg, On axisymmetric and stationary solutions of the self-gravitating Vlasov system, Classical Quantum Gravity 33, 155008 (2016).

[2] H. Andréasson, M. Kunze, and G. Rein, Existence of axially symmetric static solutions of the Einstein-Vlasov system, Commun. Math. Phys. 308, 23 (2011).

[3] H. Andréasson, M. Kunze, and G. Rein, Rotating, stationary, axially symmetric spacetimes with collisionless matter, Commun. Math. Phys. 329, 787 (2014).

[4] H. Andréasson, Sharp bounds on $2 \mathrm{~m} / \mathrm{r}$ of general spherically symmetric static objects, J. Diff. Eq. 245, 2243 (2008).

[5] H. Andréasson, Sharp bounds on the critical stability radius for relativistic charged spheres, Commun. Math. Phys. 288, 715 (2009).

[6] R. Meinel and M. Hütten, On the black hole limit of electrically counterpoised dust configurations, Classical Quantum Gravity 28, 225010 (2011).

[7] R. Meinel, Quasistationary collapse to the extreme Kerr black hole, Ann. Phys. (Berlin) 13, 600 (2004).

[8] R. Meinel, On the black hole limit of rotating fluid bodies in equilibrium, Classical Quantum Gravity 23, 1359 (2006).

[9] R. Meinel, M. Ansorg, A. Kleinwächter, G. Neugebauer, and D. Petroff, Relativistic Figures of Equilibrium (Cambridge University Press, Cambridge, England, 2012).

[10] T. Fischer, S. Horatschek, and M. Ansorg, Uniformly rotating rings in general relativity, Mon. Not. R. Astron. Soc. 364, 943 (2005).

[11] M. Ansorg, A. Kleinwächter, and R. Meinel, Relativistic Dyson rings and their black hole limit, Astrophys. J. 582, L87 (2003).

[12] V. P. Frolov, W. Israel, and W. G. Unruh, Gravitational fields of straight and circular cosmic strings: Relation between gravitational mass, angular deficit, and internal structure, Phys. Rev. D 39, 1084 (1989).

[13] S. J. Hughes, D. J. Mc Manus, and M. Vandyck, Weak-field gravity of circular cosmic strings, Phys. Rev. D 47, 468 (1993).

[14] D. J. Mc Manus and M. A. Vandyck, Weak-field gravity of revolving circular cosmic strings, Phys. Rev. D 47, 1491 (1993).

[15] H. Andréasson and G. Rein, On the steady states of the spherically symmetric Einstein-Vlasov system, Classical Quantum Gravity 24, 1809 (2007).

[16] H. Andréasson, The Einstein-Vlasov system/kinetic theory, Living Rev. Relativity 14, 4 (2011).

[17] H. Andreasson, D. Fajman, and M. Thaller, Models for self-gravitating photon shells and geons, Ann. Inst. Henri Poincaré 18, 681 (2017).
[18] A. Akbarian and M. W. Choptuik, Critical collapse in the spherically symmetric Einstein-Vlasov model, Phys. Rev. D 90, 104023 (2014).

[19] S. L. Shapiro and S. A. Teukolsky, Relativistic stellar systems with rotation, Astrophys. J. 419, 636 (1993).

[20] S. L. Shapiro and S. A. Teukolsky, Relativistic stellar systems with spindle singularities, Astrophys. J. 419, 622 (1993).

[21] J. M. Bardeen, Rapidly Rotating Stars, Disks, and Black holes, edited by C. Dewitt and B.S. Dewitt (Gordon and Breach, Science Publishers, Inc, New York, 1973), pp. 241-289.

[22] Automated Solution of Differential Equations by the Finite Element Method: The FEniCS book, edited by A. Logg, K.-A. Mardal, and G. Wells, Lecture Notes in Computational Science and Engineering Vol. 84 (Springer Science \& Business Media, Berlin, Heidelberg, 2012).

[23] A. Logg and G. N. Wells, DOLFIN: Automated finite element computing, ACM Trans. Math. Softw. 37, 1 (2010).

[24] W. Dörfler, A convergent adaptive algorithm for Poissons equation, SIAM J. Numer. Anal. 33, 1106 (1996).

[25] D. G. Anderson, Iterative procedures for nonlinear integral equations, J. ACM 12, 547 (1965).

[26] H. F. Walker and P. Ni, Anderson acceleration for fixedpoint iterations, SIAM J. Numer. Anal. 49, 1715 (2011).

[27] A. Komar, Covariant conservation laws in general relativity, Phys. Rev. 113, 934 (1959).

[28] Y. B. Zel'dovich and I. D. Novikov, Relativistic astrophysics I, Sov. Phys. Usp. 7, 763 (1965).

[29] J. M. Bardeen, W. H. Press, and S. A. Teukolsky, Rotating black holes: Locally nonrotating frames, energy extraction, and scalar synchrotron radiation, Astrophys. J. 178, 347 (1972).

[30] E. Ames, H. Andréasson, and O. Rinne (to be published).

[31] J. M. Bardeen and R. V. Wagoner, Relativistic disks. I. Uniform rotation, Astrophys. J. 167, 359 (1971).

[32] M. Ansorg and H. Pfister, A universal constraint between charge and rotation rate for degenerate black holes surrounded by matter, Classical Quantum Gravity 25, 035009 (2008).

[33] K.S. Thorne, Nonspherical gravitational collapse: A short review, in Magic Without Magic: John Archibald Wheeler, edited by J. R. Klauder (W. H. Freeman \& Co., San Francisco, 1972), pp. 231-258.

[34] T. Futamase and D. Garfinkle, What is the relation between $\Delta \varphi$ and $\mu$ for a cosmic string?, Phys. Rev. D 37, 2086 (1988).

[35] D. Garfinkle, General relativistic strings, Phys. Rev. D 32, 1323 (1985). 
[36] D. Garfinkle and P. Laguna, Contribution of gravitational self-interaction to $\Delta \varphi$ and $\mu$ for a cosmic string, Phys. Rev. D 39, 1552 (1989).

[37] A. Vilenkin, Gravitational field of vacuum domain walls and strings, Phys. Rev. D 23, 852 (1981).

[38] H. Andréasson and G. Rein, A numerical investigation of the stability of steady states and critical phenomena for the spherically symmetric Einstein-Vlasov system, Classical Quantum Gravity 23, 3659 (2006).

[39] J. L. Friedman, Ergosphere instability, Commun. Math. Phys. 63, 243 (1978).

[40] V. Cardoso, P. Pani, M. Cadoni, and M. Cavaglià, Ergoregion instability of ultracompact astrophysical objects, Phys. Rev. D 77, 124044 (2008). 